\title{
Solution for Use in Drinking Water/Milk Dosage Form
}

National Cancer Institute

\section{Source}

National Cancer Institute. Solution for Use in Drinking Water/Milk Dosage Form. NCI

Thesaurus. Code C149936.

Solution for veterinary use, intended for administration by incorporation into the animal drinking water, milk or milk replacer. If direct oral administration to individual animals is also authorized, the method of administration, including any necessary dilution, is clearly described in the product information. 